\title{
Erros de medicação: análise ergonômica de utensílios da sala de medicação em ambiente hospitalar
}

\author{
Medication errors: ergonomic analysis of the utensils in the \\ medication living in the hospital environment
}

\author{
Rosimeri Franck Pichler¹, Lucas José Garcia², Eva Maria Seitz³, \\ Giselle Schmidt Alves Díaz Merino ${ }^{4}$, Leila Amaral Gontijo5, Eugenio Andres Díaz Merino ${ }^{6}$
}

\begin{abstract}
Resumo
Da prescrição até a administração de medicamentos em pacientes podem ocorrer erros que causam dor, sofrimento e, em casos extremos, a morte. Assim, o objetivo geral deste artigo foi identificar os problemas relacionados aos utensílios da sala de preparo de medicação de um hospital da Grande Florianópolis no que tange à ergonomia física, cognitiva e organizacional, a fim de propor possíveis soluções no preparo de medicação para a eliminação ou minimização de erros no fluxo de medicação. Como método, fez-se: pesquisa bibliográfica, visita a campo e avaliação dos utensílios quanto à ergonomia física, cognitiva e organizacional. Como resultados, elaborou-se um quadro de análise de alguns utensílios da sala de medicação do hospital escola estudado, os quais foram relacionados com possíveis soluções para a otimização do processo de medicação. Assim, espera-se contribuir para a melhoria do trabalho dos profissionais da saúde e, consequentemente, promover uma melhor prestação de serviços à população.

Palavras-chave: saúde; preparações farmacêuticas; erros de medicação; engenharia humana.

Abstract

From prescription to medication administration, errors that cause pain, suffering and, in extreme cases, death may occur in patients. Thus, the aim of this paper was to identify the problems related to the utensils used in the medication room of a hospital in Florianópolis, in respect to physical, cognitive and organizational ergonomics, in order to propose possible solutions to prepare medications for disposal or minimization of errors in the stream of medication. As a method, they were used: literature review, field visit and assessment tools as physical, cognitive and organizational ergonomics. As a results, it is presented a framework analysing some utensils of medication room, which has been elaborated and related with possible solutions to optimization of the medication process. Thus, it is expected to contribute to improving the work of health professionals and promote better service to the population.
\end{abstract}

Keywords: health; pharmaceutical preparations; medication errors; human engineering.

Trabalho realizado nas dependências de um Hospital Escola na Grande Florianópolis - Florianópolis (SC), Brasil.

'Bacharel em Desenho Industrial pela Universidade Federal de Santa Maria (UFSM) - Santa Maria (RS); Mestranda em Design pela Universidade Federal do Rio Grande do Sul (UFRGS) - Porto Alegre (RS), Brasil.

${ }^{2}$ Mestre em Design pela Universidade Federal de Santa Catarina (UFSC); Doutorando em Design pela UFSC - Florianópolis (SC), Brasil.

${ }^{3}$ Mestre em Engenharia de Produção pela UFSC; Doutoranda em Engenharia de Produção pela UFSC - Florianópolis (SC), Brasil.

${ }^{4}$ Mestre em Design e Expressão gráfica UFSC; Doutoranda em Engenharia de Produção pela UFSC - Florianópolis (SC), Brasil.

${ }^{5}$ Doutora em Ergonomia pela Université Paris XIII - França; Professora Associada da UFSC - Florianópolis (SC), Brasil.

${ }^{6}$ Doutor em Engenharia de Produção pela UFSC; Professor Associado IV da UFSC - Florianópolis (SC), Brasil.

Endereço para correspondência: Rosimeri Franck Pichler - Rua Guilherme Brust, 383 - Languiru - CEP: 95890-000 - Teutônia (RS), Brasil -

E-mail: rosi.pichler@gmail.com

Conflito de interesses: nada a declarar. 


\section{INTRODUÇÃO}

A segurança do paciente em ambiente hospitalar tem se tornado uma preocupação central na área da saúde, e o erro representa um risco constante nesse contexto, já que resulta em danos físicos e psicológicos a todos os envolvidos ${ }^{1}$. Estima-se que por ano até 98 mil americanos morrem em decorrência de erros médicos, dentre os quais se enquadram os erros de medicação ${ }^{2}$. Os erros de medicação podem ser definidos como eventos evitáveis que conduzam ou causem a administração de medicação inadequada e danos ao paciente, os quais podem estar relacionados a prática profissional, produtos da saúde, procedimentos, sistemas, prescrição, comunicação, rotulagem, embalagem, dispensação, distribuição, monitoramento, uso, entre outros ${ }^{3}$.

Alguns autores ${ }^{4,5}$ salientam que os erros de medicação podem ocorrer em todas as suas etapas - aquisição, prescrição, transcrição, dispensação, administração e monitoração da resposta do paciente - , no entanto esse tipo de erro é mais comum na prescrição e na administração. Na sala de medicação do posto de enfermagem (local onde acontece o preparo de medicações antes da administração), problemas na organização e no acondicionamento de medicações são fatores diretamente relacionados à ocorrência de erros e consequentemente de danos aos pacientes ${ }^{1}$.

Erros na seleção do medicamento podem ocorrer em razão da falta de organização de armários e gavetas, ou pela distribuição de medicamentos diferentes que apresentem embalagens semelhantes. Esse tipo de erro está entre os que têm maior potencial de causar eventos adversos nos pacientes ${ }^{6} \mathrm{e}$ "podem levar a importantes agravos à saúde dos pacientes"

Dessa forma, a incidência de erros em medicação pode gerar prolongamento da internação, aumento dos custos de hospitalização, necessidade de tratamentos adicionais, exames e procedimentos extras, assim como dor, sofrimento, sequelas e a morte. Além disso, eles podem afetar não apenas o paciente mas também a organização hospitalar, denegrindo a sua imagem, gerando desconfiança e o aumento de custos, e os profissionais da saúde, que sofrem consequências administrativas (demissões) e psicológicas (culpa).

Com base nesse panorama, o presente artigo teve como objetivo identificar os problemas relacionados aos utensílios da sala de preparo de medicação de um hospital da Grande Florianópolis, no que tange à ergonomia física, cognitiva e organizacional, com o intuito de reduzir os riscos de erros em medicação.

\section{Ergonomia no contexto hospitalar}

O funcionamento de um hospital envolve diversos tipos de profissionais em turnos contínuos (da área da saúde e outras áreas), suprimentos perecíveis, equipamentos sofisticados, programação de horários de medicamentos, turnos de trabalho, visitantes, entre outros inúmeros fatores. Nesse sentido, a complexidade do funcionamento de um hospital pode ser comparada ao de uma empresa industrial ${ }^{9}$. Essa complexidade, por sua vez, exige uma gama de soluções e sistemas integrados que funcionem como aliados na humanização dos serviços prestados no ambiente hospitalar ${ }^{10}$.

Os avanços tecnológicos têm auxiliado tanto a prática como a formação dos profissionais de saúde, porém seu uso não pode resultar no esquecimento de quem é o objeto principal da questão, o ser humano. Assim, os autores salientam que a valorização dos elementos técnicos sobre os humanísticos e a consequente desumanização da formação dos profissionais da saúde torna o estudo da humanização, nesse âmbito, atual e necessário ${ }^{11}$.

A Ergonomia apresenta como domínios de especialização: a ergonomia física, cognitiva ou organizacional. A ergonomia física trata da relação das características anatômicas, antropométricas, fisiológicas e biomecânicas do homem com a atividade física realizada, envolvendo, por exemplo, o estudo do posto de trabalho, posturas, alcances, distúrbios, entre outros. A ergonomia cognitiva compreende os processos mentais (percepção, memória, raciocínio) nas interações do homem com os elementos de um sistema, envolvendo o estudo das cargas de trabalho, estresse, desempenho, entre outros. Por fim, a ergonomia organizacional busca a melhoria do sistema como um todo (estrutural, política e processual), incluindo o estudo das comunicações, cooperatividade, participação, gestão da qualidade, entre outros ${ }^{9,12}$. A atuação da Ergonomia na área da saúde pode ser denominada de Ergonomia Hospitalar que, mesmo pouco difundida, apresenta pesquisas significativas na área de equipamentos médicos e procedimentos ${ }^{13}$.

Por fim, autores ${ }^{14}$ argumentam que a integração entre as diversas áreas de conhecimento, como a Acessibilidade, a Antropometria, o Design Ergonômico, o Design Universal, a Ergonomia e a Usabilidade, corroboram para o emprego de soluções mais condizentes com as reais necessidades dos usuários, permitindo contemplar as diversas potencialidades, as quais não seriam adequadamente atendidas sob uma única ótica. Portanto, a Ergonomia e a Usabilidade são consideradas fontes ricas de conceitos e métodos que permitem a concepção ou a correção de projetos de forma harmoniosa com o sistema de saúde ${ }^{15,16}$.

\section{Erros de medicação em ambiente hospitalar}

O erro é comumente entendido como a ocorrência desviada do padrão estabelecido, ou seja, a atividade conforme descrita não foi executada corretamente, por algum motivo, e não atingiu seu objetivo ${ }^{9,17,18}$. O erro de medicação corresponde a qualquer 
evento que possa levar ao uso inadequado de medicamentos e que possa ser evitado, podendo ou não, lesar o paciente. Esse tipo de erro pode estar relacionado a prática profissional, produtos, procedimentos, problemas de comunicação, prescrição, transcrição, dispensação, distribuição, embalagens, rótulos, administração, monitoramento do paciente, entre outros ${ }^{7,19}$.

$\mathrm{O}$ aumento do risco de erro está associado à ocorrência de variação de comportamento além do aceitável para determinada tarefa; assim, quando os limites dessa variação são ultrapassados, aumenta-se o risco de acidentes ${ }^{20}$. Essa variação de comportamento, por sua vez, é causada por fatores internos (indivíduo) e externos (ambiente).

A divisão mais básica de erro é entre lapso e engano. O lapso ocorre quando o usuário sabe executar a tarefa, mas acidentalmente alguma coisa ocorre errada durante a realização dela. Já o engano ocorre quando o usuário tem o conhecimento errado da execução da tarefa e por isso comete erros. Quanto às causas, o lapso está relacionado à dinâmica mental automática e é causado por cansaço, ansiedade, ruído ou outros fatores que desviam a atenção do indivíduo. O engano, por sua vez, resulta de processos elaborados de resolução de problemas, podendo ocorrer erros por falta de conhecimento (engano baseado no conhecimento) ou por má interpretação do problema a ser resolvido (engano baseado em regras) ${ }^{21,22}$.

Assim, percebe-se que o erro está associado tanto a processos cognitivos humanos como também aos aspectos ambientais e ergonômicos. Para tanto, o erro pode ser analisado do ponto de vista do sistema e das pessoas ${ }^{23}$. Quando se centraliza o sistema, julga-se que as pessoas falharam por causa de erros no processo, sendo o erro uma consequência e não uma causa. Nessa visão, não importa quem o cometeu, mas sim o que levou alguém a cometê-lo. Quando centrado nas pessoas, o erro está relacionado à desatenção, desmotivação, negligência ou imprudência, focando as medidas para o controle do comportamento humano e de atitudes morais. Os sistemas modernos e complexos atuais corroboram para o aumento da incidência de erros humanos ${ }^{24}$. O ambiente hospitalar, por se tratar de um sistema complexo de informações e inter-relações, torna-se um local propício à ocorrência de erros, tanto de sistemas como de pessoas.

\section{METODOLOGIA}

Para o desenvolvimento da pesquisa, utilizou-se em um primeiro momento, como procedimento técnico, o levantamento bibliográfico e, em um segundo momento, realizou-se visitas de campo às dependências de um hospital escola localizado na Grande Florianópolis, que atua desde $1980^{25,26}$.

A estrutura desse hospital compreende quatro áreas básicas (clínica médica, cirúrgica, pediatria e tocoginecologia), além do atendimento 24 horas, que atende por dia a média de 400 pacientes. O foco do trabalho compreendeu as unidades de internação tanto cirúrgica quanto médica. Cada unidade acomoda o número de 30 leitos, distribuídos em 15 quartos com 2 leitos cada. O número de profissionais por unidade é em torno de 6, sendo 4 técnicos em Enfermagem, 1 enfermeiro diurno e 1 enfermeiro noturno. Assim, cada técnico em Enfermagem é responsável pela administração da medicação e atende em média a 7 pacientes por dia. Durante essas visitas, foi realizada uma observação assistemática, entrevistas semiestruturadas, além de registros fotográficos e de áudio para posterior análise e registro de informações ${ }^{27}$.

A partir dos dados coletados, realizou-se uma análise qualitativa e elaborou-se um quadro indicativo dos problemas encontrados nos utensílios utilizados na etapa de preparo de medicamentos na sala de medicação. Os problemas identificados foram divididos conforme os domínios da ergonomia física, cognitiva e organizacional. Os procedimentos metodológicos adotados na pesquisa podem ser visualizados na Figura 1.

A presente pesquisa é composta por uma equipe multidisciplinar e faz parte de um projeto maior, denominado "Erro humano na saúde: o caso com medicamentos de alto risco por via intravenosa", aprovado segundo os critérios do Comitê de Ética em Pesquisa com Seres Humanos da Universidade Federal de Santa Catarina, em outubro de 2013, sob número 20248813.8.0000.0121.

\section{RESULTADOS E DISCUSSÃO}

Com base nas observações realizadas durante as visitas a campo, foi possível descrever o fluxo de medicação do hospital em estudo, que envolve o trajeto do medicamento desde sua prescrição até a administração no paciente. Assim, o fluxo de medicação compreende as seguintes etapas (Figura 2):

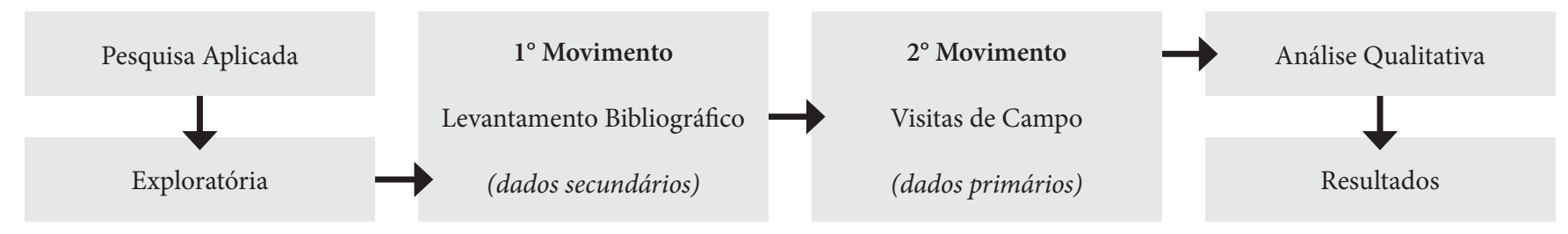

Figura 1. Procedimentos metodológicos da pesquisa 
- Prescrição: realizada pelo médico no Posto de Enfermagem, a partir do diagnóstico obtido com o paciente.

- Dispensação: compreende, primeiramente, a conferência das prescrições pelo farmacêutico quanto ao nome do medicamento, a dosagem e a via de administração (oral, intravenosa, tópica, entre outras). Após essa conferência, o dispensador (bolsista de qualquer habilitação) realiza a leitura da prescrição e a separação dos medicamentos correspondentes. Por fim, o escriturário (bolsista de qualquer habilitação) realiza novamente a leitura da prescrição e confere com o medicamento separado pelo dispensador.

- Preparação: é fase de organização dos medicamentos e ocorre na sala de medicação. O escriturário coloca a prescrição em uma prancheta deixada no balcão da sala de medicação, e os medicamentos são guardados nos escaninhos correspondentes ao quarto e leito do paciente. O técnico em Enfermagem, quando chega para o seu turno de trabalho, transcreve as informações das prescrições de seus pacientes para pequenos pedaços de papel, denominadas as cinco certezas: nome do paciente (1), quarto e leito do paciente (2), nome do medicamento (3), dosagem e via (4), e hora da administração da medicação no paciente (5). Aproximando-se da hora da administração dos

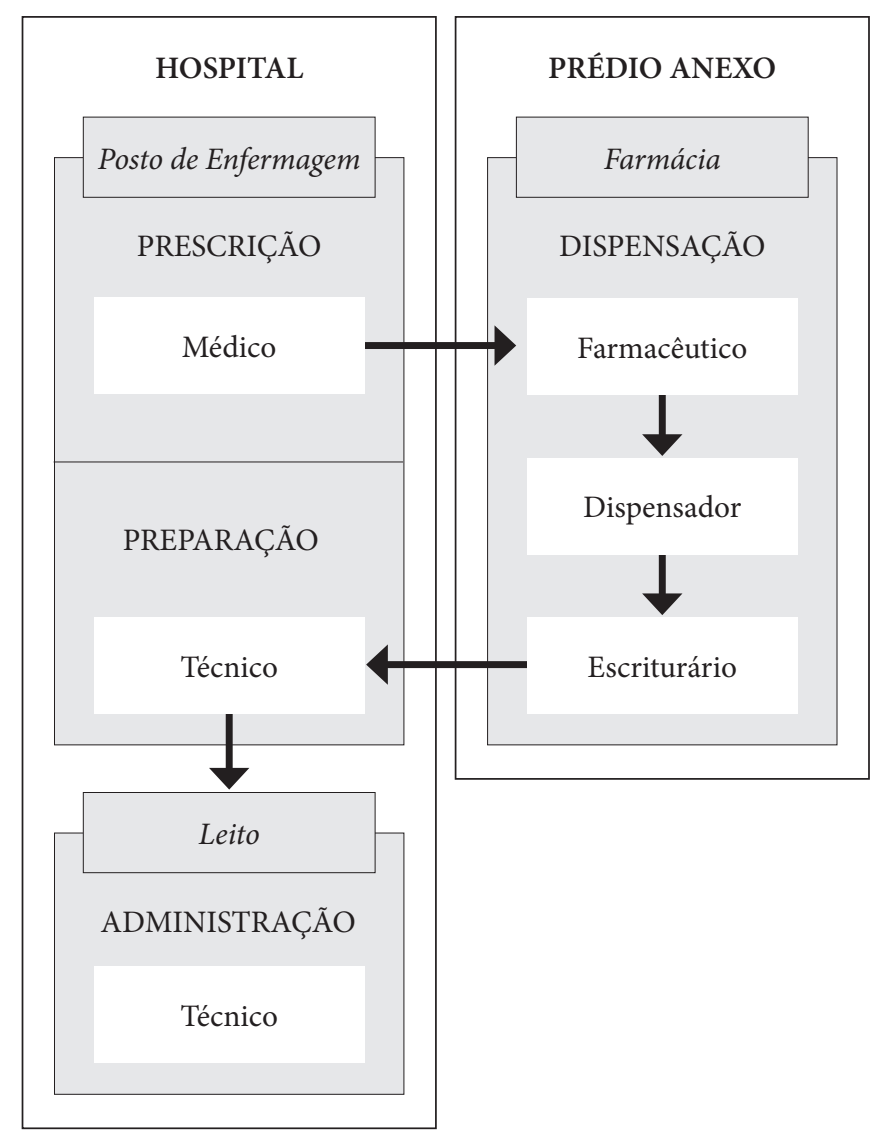

Figura 2. Fluxo de medicação medicamentos, o técnico em Enfermagem prepara e organiza os medicamentos em uma bandeja. Nesse momento, são realizadas as três leituras do nome do medicamento: quando se retira do escaninho, enquanto se prepara e quando a embalagem é descartada. Assim, na bandeja são colocados os medicamentos lado a lado, posicionando abaixo de cada grupo de medicamento o papelzinho com as cinco certezas.

- Administração: é o momento da medicação; o técnico em Enfermagem, antes de realizar a administração, faz a conferência dos dados do paciente na cabeceira do leito, com as informações presentes no papelzinho. O medicamento é então administrado, finalizando o fluxo de medicação.

Como problemas encontrados na etapa de preparação, destacam-se:

- falta de alertas do sistema na ocorrência de erro;

- falta de conhecimento sobre os medicamentos e desatualização quanto aos princípios ativos dos medicamentos;

- falta de controle e padronização de procedimentos no preparo de medicamentos;

- similaridade de formas, nomenclaturas e cores das embalagens dos medicamentos;

- dificuldade de encontrar as informações nos rótulos e nas embalagens de medicamentos em decorrência da falta de hierarquização das informações e ilegibilidade;

- ambiente de armazenamento dos medicamentos inapropriado e mal organizado;

- a prescrição eletrônica já foi implantada há 15 anos, porém não são utilizadas todas as suas potencialidades para agilizar e tornar o processo mais seguro;

- perda de informações dos medicamentos ao longo do processo (adesivos com baixa legibilidade e incompletos).

Esses aspectos podem ser corroborados por alguns autores ${ }^{1}$ que explicam que na sala de medicação do posto de enfermagem existem problemas na organização e no acondicionamento de medicações. Os autores reforçam que esses fatores estão diretamente relacionados à ocorrência de erros e danos aos pacientes, e que erros na seleção do medicamento podem ocorrer em razão da falta de organização de armários e gavetas ou pela distribuição de medicamentos diferentes que apresentam embalagens semelhantes.

Mediante esses problemas, inicialmente, foram estudados os utensílios que envolvem os processos de preparo de medicamentos na sala de medicação, são eles: o escaninho (Figura 3A), local de armazenamento dos medicamentos para realização do preparo e organização da bandeja; o papel (Figura 3B), ticket com informações dos pacientes, medicamentos e procedimentos de administração; uma gaveta (Figura 3C), local em que são 
depositados os medicamentos que sobraram das medicações efetuadas; as embalagens dos medicamentos (Figura 3D), ampolas, comprimidos, entre outros; e a bandeja (Figura 3E), utensílio de transporte dos medicamentos preparados até o quarto do paciente.

Esses utensílios foram organizados em forma de quadro e analisados segundo os aspectos da ergonomia física, cognitiva e organizacional (Quadro 1).

Com relação ao escaninho, observa-se a predominância de problemas no que tange aos aspectos físicos, os quais podem ser minimizados com o desenvolvimento de um novo produto que favoreça as tarefas executadas nessa atividade, por meio do desenvolvimento de mobiliários específicos para a atividade, com alturas fixas e compartimentos que impeçam a mistura de medicamentos dos pacientes, além da padronização das informações, com uma adequada hierarquização delas, facilitando assim a rápida identificação pelos funcionários, evitando enganos na armazenagem e retirada de medicamentos e diminuindo o estresse e o tempo de realização da atividade.
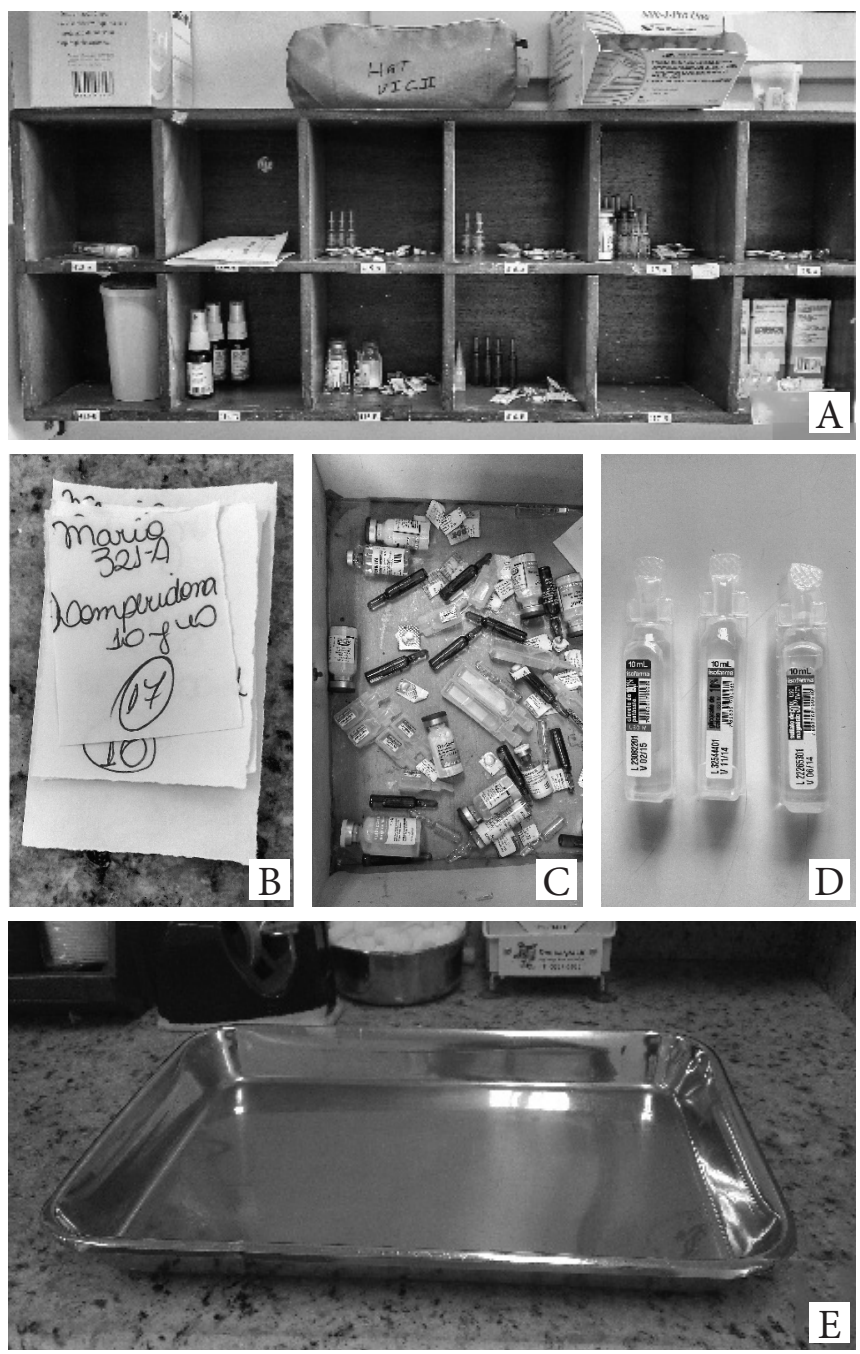

Figura 3. Utensílios da sala de medicação
Quanto aos aspectos cognitivos e organizacionais, estes devem ser solucionados a partir de ações que promovam a melhoria do ambiente de trabalho, como treinamentos e períodos de adaptação do funcionário à atividade.

No utensílio papel, os problemas decorrem da questão organizacional de falta de padronização do processo, ou seja, da inexistência de um modelo para o preenchimento das informações que seja de conhecimento de todos os envolvidos e que facilite a visualização das informações.

Quanto à bandeja, os principais problemas circundam a ergonomia física e cognitiva sobretudo no que se relaciona ao preparo e transporte dos medicamentos. Além da sobrecarga mental que o próprio preparo do medicamento impõe ao profissional responsável, a configuração do utensílio não auxilia a atividade, já que os medicamentos de vários pacientes são dispostos muito próximos e com uma identificação pouco aparente. Além disso, a preocupação com a segurança dos medicamentos no transporte até o leito é mais um item que pode intensificar o estresse mental do indivíduo. Assim, a aquisição de um utensílio específico para o armazenamento, a organização e o transporte dos medicamentos é crucial para a minimização de erros nessa etapa.

Os aspectos organizacionais são os principais causadores dos problemas encontrados no utensílio gaveta. Mesmo que os problemas de ordem física e cognitiva sejam solucionados ou minimizados, enquanto os profissionais não passarem a utilizar a gaveta da forma correta, os medicamentos continuarão sendo perdidos por causa da falta de gestão dessas sobras. Essa questão pode ser solucionada com treinamento e conscientização dos profissionais. Da mesma forma, a solução de problemas de ordem cognitiva e física pode servir de estímulo para que os profissionais utilizem de forma correta esse utensílio.

No que tange às embalagens dos medicamentos, o principal problema observado é a semelhança entre embalagens de mesma via, seguido da falta de padronização em relação à hierarquização das informações. Em relação às ampolas de vidro, especificamente, observa-se a falta de legibilidade de todas as informações impressas. Quanto às drágeas, embora haja diferenças significativas entre as embalagens e os comprimidos, a necessidade de transcrição das informações da embalagem para adesivos colados no momento da dispensação dificulta a diferenciação dos medicamentos além de apresentar problemas de ilegibilidade em razão da qualidade de impressão.

Assim, observa-se que as condições físicas dos ambientes existentes no hospital analisado, nos quais os trabalhadores estão inseridos, favorecem a ocorrência do erro. Conforme já observado anteriormente, o erro não deve ser visto como um indivíduo cometendo um erro, mas como uma condição projetada que permitiu a execução de uma ação errada. 
Quadro 1. Análise dos utensílios com relação aos domínios da Ergonomia

\begin{tabular}{|c|c|c|c|}
\hline & Ergonomia física & Ergonomia cognitiva & Ergonomia organizacional \\
\hline Escaninho & $\begin{array}{c}\text { Ilegibilidade } \\
\text { Altura fixa } \\
\text { Baixa luminosidade/contraste } \\
\text { Falta de divisões por tipo de medicamento } \\
\text { Espaço limitado } \\
\text { Exige precisão de movimentos do } \\
\text { funcionário }\end{array}$ & $\begin{array}{l}\text { Poluição visual } \\
\text { Confusão mental }\end{array}$ & $\begin{array}{c}\text { Desorganização do espaço } \\
\text { Exposição inapropriada dos } \\
\text { medicamentos }\end{array}$ \\
\hline Papel & $\begin{array}{l}\text { Ilegibilidade } \\
\text { Formatos e cores não auxiliam a função }\end{array}$ & $\begin{array}{l}\text { Confusão mental } \\
\text { Erros de transcrição } \\
\text { Inseguro }\end{array}$ & Falta de padronização \\
\hline Bandeja & $\begin{array}{c}\text { Falta de divisórias } \\
\text { Falta proteção aos medicamentos } \\
\text { Ausência de pegas } \\
\text { Instável } \\
\text { Espaço limitado }\end{array}$ & $\begin{array}{l}\text { Confusão mental } \\
\text { Enganos } \\
\text { Estresse }\end{array}$ & $\begin{array}{l}\text { Exposição inapropriada dos } \\
\text { medicamentos }\end{array}$ \\
\hline Gaveta & $\begin{array}{c}\text { Falta de divisórias } \\
\text { Uso de materiais que dificultam a } \\
\text { higienização } \\
\text { Dificuldade de acesso }\end{array}$ & $\begin{array}{l}\text { Mistura dos medicamentos } \\
\text { favorece o engano } \\
\text { Estresse e pressão do tempo }\end{array}$ & $\begin{array}{c}\text { Falta de higiene } \\
\text { Falta de controle de conteúdo } \\
\text { Fluxo de medicamentos interrompido } \\
\text { Falta de gestão na separação dos } \\
\text { medicamentos bons dos vencidos } \\
\text { Exposição inapropriada dos } \\
\text { medicamentos } \\
\text { Perda de medicamentos }\end{array}$ \\
\hline $\begin{array}{l}\text { Embalagem de } \\
\text { medicamentos }\end{array}$ & $\begin{array}{c}\text { Semelhança de cores } \\
\text { Semelhança de formatos } \\
\text { Semelhança de nomenclatura } \\
\text { Ilegibilidade }\end{array}$ & $\begin{array}{l}\text { Nomes extensos de difícil } \\
\text { memorização }\end{array}$ & $\begin{array}{l}\text { Necessidade de transcrição das } \\
\text { informações (drágeas) }\end{array}$ \\
\hline
\end{tabular}

\section{CONSIDERAÇÕES FINAIS}

Ao final desta pesquisa, observou-se que a aplicação dos conceitos da Ergonomia são eficazes na identificação de problemas complexos como os vivenciados em âmbito hospitalar. Portanto, a realização de visitas a campo, observando a realização das atividades, compreende uma importante ferramenta para diagnosticar e detectar os pontos de fragilidade do sistema.

Com base no levantamento realizado no local, foi possível identificar os principais problemas relacionados à má realização das atividades na sala de medicação, que podem, de alguma forma, induzir o profissional ao erro. Com a elaboração do quadro indicativo de problemas quanto à ergonomia física, cognitiva e organizacional, buscou-se identificar os possíveis pontos de ação para a minimização de erros no ambiente hospitalar, especificamente na etapa de preparação dos medicamentos. Assim, foram apresentadas possíveis soluções, sejam elas para projeto do ambiente de trabalho, para melhorias quanto às cargas mentais de trabalho ou quanto ao sistema, atuando na melhoria da gestão do serviço oferecido como um todo.

Como estudos futuros, estima-se a continuação da fase de diagnóstico a fim de obter dados quantificáveis, além de propor e desenvolver algumas das soluções aqui identificadas, testando a efetividade dos resultados quanto à minimização de erros no processo de medicação. Assim, espera-se que tais estudos venham a promover melhorias na área da Saúde, tanto para os profissionais da saúde quanto para o ambiente e o sistema hospitalar como um todo, impactando em uma prestação de serviço de melhor qualidade à população.

\section{AGRADECIMENTOS}

Agradecemos à Coordenação de Aperfeiçoamento de Pessoal de Nível Superior (CAPES), ao Conselho Nacional de Desenvolvimento Científico e Tecnológico (CNPq), à Fundação de Amparo à Pesquisa e Inovação do Estado de Santa Catarina (FAPESC), ao Programa de Pós-graduação em Design da Universidade Federal de Santa Catarina (PPGD/UFSC), ao Programa de Pós-graduação em Design da Universidade Federal do Rio Grande do Sul (PGDesign/UFRGS), ao Programa de Pós-graduação em Engenharia de Produção da UFSC (PPGEP), ao hospital que concedeu a oportunidade para o desenvolvimento desta pesquisa e ao Núcleo de Gestão de Design e Laboratório de Design e Usabilidade da UFSC (NGD-LDU/UFSC). 


\section{REFERÊNCIAS}

1. Raduenz AN, Hoffmann P, Radunz V, Dal Sasso GTM, Maliska ICA, Marck PB. Cuidados de enfermagem e segurança do paciente: visualizando a organização, acondicionamento e distribuição de medicamentos com método de pesquisa fotográfica. Rev Latino-Am Enfermagem. 2010:18(6):1045-54.

2. Lesar TS. Medication errors: creating and selecting safer drug products. Clin Oncol. 2008:3(3):1-8

3. NCCMERP - National Coordinating Council for Medication Error Reporting and Prevention. 2013 [Cited 2013 Nov. 01]. Available from: http://www.nccmerp.org/public/aboutmederror.html

4. National Research Council. Preventing medication erros: quality chasm series. Washington: National Academies Press; 2007.

5. Meller RD, Pazour JA, Thomas LM, Mason SJ, Root SE, Churchill WW. Third-party repackaging in hospital pharmacy unit dose acquisition. Am J Health Syst Pharm. 2010:67(13):1108-14.

6. Kaushal R, Bates DW, Landrigan C, McKenna KJ, Clapp MD, Federico F, et al. Medication errors and adverse drug events in pediatric inpatients. JAMA. 2001;285(16):2114-20.

7. Anacleto TA, Rosa MB, Neiva HM, Martins MAP. Erros de medicação. Farmácia Hospitalar. Pharmacia Brasileira. 2010;1:1-24.

8. Kohn LT, Corrigan JM, Donaldson MS. To err is human: building a safer health system. Washington: National Academy Press; 2000.

9. Iida I. Ergonomia: projeto e produção. $2^{\text {a }}$ ed. revisada e ampliada. São Paulo: Edgar Blücher; 2005.

10. Skrabe C. Chegou a hora e a vez do design. In: Anuário de Design Hospitalar. Anuário Hospital Best - Eximia Comunicação. 2010 [Cited 2013 Oct. 14]. Avaliable from: http://issuu.com/eximia/docs/anuario2010.

11. Silva MR, Sakamoto J, Gallian DMC. A cultura estética e a educação do gosto como caminho de formação e humanização na área da saúde. Trab Educ Saúde [online]. 2014;12(1):15-28.

12. ABERGO. Associação Brasileira de Ergonomia. O que é Ergonomia. 2013 [cited 2013 Oct. 03]. Available from: http://www.abergo.org.br/ internas.php?.pg=o_que_e_ergonomia.

13. Cardoso VMB, Moraes A. Ergonomia hospitalar: atividades de alimentação e deficiências dos equipamentos disponíveis. In: ENEGEP, 1998, Rio de Janeiro.
14. Lucio CC, Paschoarelli LC. Usabilidade e acessibilidade de equipamentos médico-hospitalares: um estudo de caso com pacientes obesos. In: Design e ergonomia: aspectos tecnológicos. São Paulo: Cultura Acadêmica; 2009.

15. Reid P, Compton WD, Grosmann JH, Fanjiang G. Building a better delivery system: a new engineering/health care partnership. Washington: The National Academy Press; 2005.

16. Serranheira F, Uva AS, Sousa P. Ergonomia hospitalar e segurança do doente: mais convergências que divergências. Rev Port Saúde Pública. 2010;10:58-73.

17. Reason J. Human error. Cambridge: Cambridge University Press; 1990.

18. Pedrassani EL. Método para registro, análise e controle de falhas humanas na manutenção de centrais elétricas [Dissertação de mestrado]. Florianópolis: Universidade Federal de Santa Catarina; 2000.

19. ANVISA. Erro de Medicação: caracterização. Agência Nacional da Vigilância Sanitária (probabilistic record linkage) [Internet]. 2013 [Cited 2013 Oct. 03]. Available from: http://s.anvisa.gov.br/wps/s/r/np.

20. Sell I. Projeto do trabalho humano: melhorando as condições de trabalho. Florianópolis: Ed. UFSC; 2002.

21. Jordan PW. An Introduction to Usability. London: Taylor \& Francis; 1998.

22. Leape LL, Bates DW, Cullen DJ, Cooper J, Demonaco HJ, Gallivan T, et al. Systems analysis of adverse drug events. JAMA. 1995;274(35):35-43.

23. Reason J. Human error models and management. West J Med. 2000;172(6):393-6.

24. De Keyser V. O Erro Humano. In: Castilho J, Villena J. Ergonomia: conceitos e métodos. Lisboa: Dinalivro; 2005.

25. Lakatos EM, Marconi MA. Fundamentos de metodologia científica. São Paulo: Atlas; 1993.

26. Gil AC. Como Elaborar Projetos de Pesquisa. São Paulo: Atlas; 2010.

27. Rutter M, Abreu SA. Pesquisa de mercado. São Paulo: Ática; 1994.

Recebido em: 22/09/2014 Aprovado em: 19/10/2014 